\title{
Primary fallopian tube carcinoma: review of MR imaging findings
}

\author{
Filipe Veloso Gomes $^{1,2}$ • João Lopes Dias ${ }^{3,4} \cdot$ Rita Lucas $^{5}$ • Teresa Margarida Cunha ${ }^{6}$
}

Received: 17 February 2015 /Revised: 20 May 2015 / Accepted: 15 June 2015 /Published online: 7 July 2015

(C) The Author(s) 2015. This article is published with open access at Springerlink.com

\begin{abstract}
Objectives To review the epidemiological and clinical features of primary fallopian tube carcinoma (PFTC), and to illustrate the spectrum of MRI findings, with pathological confirmation.

Methods This article reviews the relevant literature on the epidemiological, clinical, and imaging features of primary fallopian tube carcinoma, with pathological confirmation, using illustrations from the authors' teaching files.

Results Primary fallopian tube carcinoma came under focus over the last few years due to its possible role on the pathogenesis of high-grade serous epithelial ovarian and peritoneal cancers. Typical symptoms, together with the presence of some of the most characteristic MRI signs, such as a
\end{abstract}

Filipe Veloso Gomes

fvgomes@gmail.com

João Lopes Dias

joaolopesdias85@gmail.com

Rita Lucas

ritalucas1@gmail.com

Teresa Margarida Cunha

tmargarida@gmail.com

1 Centro Hospitalar do Algarve, Faro, Portugal

2 Department of Biomedical Sciences and Medicine, Regenerative Medicine Program, University of Algarve, Faro, Portugal

3 Department of Radiology, Hospital de S.José, CHLC, Lisbon, Portugal

4 Nova Medical School/Faculdade de Ciências Médicas, Lisbon, Portugal

5 Department of Radiology, Hospital de Sto. António dos Capuchos, CHLC, Lisbon, Portugal

6 Department of Radiology, Instituto Português de Oncologia de Lisboa Francisco Gentil, Lisbon, Portugal "sausage-shaped" pelvic mass, hydrosalpinx, and hydrometra, may signal the presence of primary fallopian cancer, and allow the radiologist to report it as a differential diagnosis.

Conclusions Primary fallopian tube carcinoma has a constellation of clinical symptoms and magnetic resonance imaging features, which may be diagnostic. Although these findings are not present together in the majority of cases, radiologists who are aware of them may include the diagnosis of primary fallopian tube cancer in their report more frequently and with more confidence.

Teaching Points

- PFTC may be more frequent than previously thought

- PFTC has specific clinical and MRI characteristics

- Knowledge of typical PFTC signs enables its inclusion in the differential diagnosis

- PFTC is currently staged under the 2013 FIGO system

- PFTC is staged collectively with ovarian and peritoneal neoplasms

Keywords Fallopian tube neoplasms · Magnetic resonance imaging $\cdot$ High-grade serous carcinoma $\cdot$ Epithelial ovarian cancer P Peritoneal cancer
Abbreviations
PFTC Primary Fallopian Tube Carcinoma
FIGO International Federation of Gynecology and Obstetrics
MRI Magnetic Resonance Imaging
CT Computed Tomography
EOC Epithelial Ovarian Cancer
T1WI T1 Weighted Images
T2WI T2 Weighted Images
DWI Diffusion Weighted Images 


\section{Introduction}

Primary fallopian tube carcinoma (PFTC) has been described as one of the rarest malignancies of the female genital tract, accounting for around $1 \%$ of all gynaecologic malignancies, occurring predominantly in post-menopausal women at a mean age of 55 years [1-7].

PFTC has come under focus in recent years, particularly in pathology and oncology scientific literature, given the likely role on the pathogenesis of ovarian cancer. This may have led to underestimate the true incidence of PFTC as ovarian cancer in the past, with significant clinical impact on the management of these patients [1, 8-12]. Several papers have emerged supporting the theory that high-grade serous ovarian carcinoma, as well as peritoneal carcinoma, may in fact originate from occult high-grade serous carcinoma in the fallopian tubes. The fimbriated ends of fallopian tubes could be the dominant site of origin, particularly in high-risk BRCA mutation carriers [7, 9, 11, 13-19].

PFTC is usually not suspected pre-operatively or even intra-operatively due to its nonspecific clinical and surgical presentation, particularly when disseminated [1, 3, 20-22]. Regarding imaging studies, PFTC is also rarely diagnosed in pre-operative studies due to nonspecific findings and overlap with ovarian cancer features [2, 3, 21-27].

The purpose of this study is to review the epidemiological and clinical features of PFTC, and to illustrate the spectrum of MRI findings, with pathological correlation.

\section{Epidemiology}

PFTC is considered a rare and aggressive type of tumour, representing $0.14-1.8 \%$ of the total gynaecological malignancies $[1,5,28]$. The incidence of PFTC may be on the rise for reasons that have not been completely understood. Associations between PFTC and socio-economic status and occupation have been described, with women of higher social classes and education being at greater risk [5, 29].

\section{Clinical presentation}

The aetiology of this type of tumour has not been completely explained. Hormonal, reproductive, and genetic factors may play a role, along with the presence of chronic inflammation of the pelvis [28].

PFTC may have a constellation of characteristic symptoms, namely colicky abdominal or pelvic pain and adnexal mass, relieved by intermittent, profuse, serosanguineous vaginal discharge, which constitute Laztko's triad (seen in only $15 \%$ of patients). Hydrops tubae profluens is a syndrome characterized by the relief of pain and shrinkage of the abdominal or pelvic mass by a vaginal discharge, which is caused by filling and emptying of a sub-occluded fallopian tube (seen in only $5 \%$ of patients). Typical symptoms, however, occur in only a minority of patients with PFTC, and most women present with less specific symptoms at the time of diagnosis [1, 28, 30-33]. The age of presentation is commonly between 40 and 60 years, with a mean age of 55 years [28]. The pre-operative diagnosis of PFTC is rarely performed, with clinical signs and symptoms pointing towards the more frequently occurring ovarian cancer or pelvic inflammatory disease. Tumour markers, particularly CA-125, have no role in the diagnosis of PFTC. Elevated CA-125 levels are, nevertheless, indicative of poor prognosis, and can be used during follow-up, as a marker of disease recurrence [34].

PFTC should be considered in the differential diagnosis of patients with postmenopausal bleeding with negative diagnostic curettage, cervical smear with intermittent suspicious abnormalities, and unexplained persistent vaginal discharge $[28,30]$.

\section{Pathological diagnosis}

Pathology remains the mainstay for diagnosis of PFTC. Serous carcinoma of the fallopian tube is the most common histological type of tubal carcinoma. It is an invasive tumour growing in papillary, glandular, and solid patterns with high grade nuclear atypia. These tumours are identical to their ovarian counterparts.

The second most common type of tumour is the endometrioid carcinoma, followed by undifferentiated, clear cell, mucinous, and transitional carcinomas [35].

PFTC is graded according to its differentiation and extent of solid components, with most tumours being poorly differentiated $[4,36]$.

Initial diagnostic criteria for the diagnosis of PFTC were proposed by Hu et al. in 1950 [37]. These were revised later by Sedlis et al. [38, 39] and currently include the following: 1 . The main tumour arises from the endosalpinx; 2 . The histological pattern reproduces the epithelium of the tubal mucosa; 3. The transition from benign to malignant tubal epithelium is demonstrable; 4 . The ovaries or endometrium are either normal or contain a tumour that is smaller than the tumour in the tube [28, 31]. Recently, Singh et al. [40], proposed an approach to the pathological assignment of primary site in high-grade serous tubal, ovarian, and peritoneal carcinoma. According to Singh, the identification of tumour inside the fallopian tubes, even in the presence of larger tumours in other localizations, supports the diagnosis of PFTC [40].

Dissemination of PFTC occurs through the transcoelomic route with implantation of cells throughout the abdominal cavity, similarly to ovarian cancer, and also through continuity to adjacent organs, transluminal migration, 
haematogenous, and lymphatic spread. Distant metastases are more common in PFTC than ovarian cancer [28, 36, 41], but a biopsy of such lesions would not distinguish them with certainty [41].

\section{Staging}

The currently accepted staging system for PFTC was developed by the International Federation of Gynecology and Obstetrics (FIGO), and currently PFTC, ovarian, and peritoneal cancers are staged collectively within the same system, although occasionally it may be impossible to attribute the tumour to a primary site [42]. Table 1 outlines the 2013 FIGO staging classification for cancer of the ovary, fallopian tube, and peritoneum.

\section{Treatment}

Current management of PFTC follows the same guidelines as ovarian cancer in terms of surgical staging, debulking, and adjuvant chemotherapy [43]. The main goal is to decrease tumour load surgically. The surgical approach consists of total abdominal hysterectomy, bilateral salpingo-oophorectomy, and infra-colic omentectomy, appendicectomy, peritoneal washings, and peritoneal biopsies [43, 44].

Routine pelvic and para-aortic lymphadenectomy is considered to be essential by some authors due to the strong likelihood of lymphatic spread. However, this issue remains controversial, and others believe that retroperitoneal node sampling and dissection suffices [28, 45].

Postoperatively, chemotherapy plays an important role in the management of early-stage PFTC, usually with platinumbased combination chemotherapy. The efficacy of current chemotherapy regimens has led to the abandonment of radiotherapy as a treatment option for PFTC due to its poor results and serious complications $[1,28]$.

Hormonal therapies may be of value in the future, given the sensitivity and response of the fallopian tube epithelium to hormonal fluctuations, although there are no current recommendations $[1,28,45]$.

\section{Prognosis}

The main prognostic factors identified for increased survival include stage, age, and residual tumour after surgery, serous subtype, and elevated pre-treatment CA-125 [28, 36]. The presence of specific symptoms may lead to an earlier diagnosis and, consequently, improved survival.

Serous PFTC in advanced stage may have a better survival than its ovarian or peritoneal counterparts [43, 46].
Earlier stage PFTC disease could also have better survival rates, given the possibility of presenting earlier and the established role of lymphadenectomy in its management. Other authors have suggested that survival outcomes are similar between PFTC and ovarian cancer, in support of identical therapeutic approaches for both types of tumours [47], and some report better survival in ovarian cancer patients compared to equivalent stage patients with PFTC. The 5-year survival rate of PFTC ranges between 22 and $57 \%$ [28].

Recurrent disease, which occurs on average 2 to 3 years after initial treatment, is associated with a dismal prognosis due to the lack of alternative treatments available [28].

\section{Imaging PFTC}

Imaging diagnosis of PFTC is important because it can help planning adequate initial surgery and avoid secondlook laparotomy. Imaging findings of PFTC are usually nonspecific, and a tubo-ovarian abscess or ovarian tumour may appear to be the most likely diagnosis given their higher prevalence [26].

Similarly to other gynaecologic tumours, the imaging approach includes ultrasound as the initial modality, followed by MRI for undetermined or suspicious adnexal masses and computed tomography (CT) or MRI for complete staging. CT is only used for staging purposes and not for pelvic mass characterization due to low soft-tissue contrast in comparison to MRI $[2,48]$.

On greyscale ultrasound, PFTC may be suspected in the presence of a tubular-shaped mass (or sausage-shaped) or a lobular mass with a cogwheel pattern [49]. On Doppler ultrasound, a low-impedance flow within the solid components may be a clue [50]. Variability of imaging characteristics in serial imaging may also point towards PFTC [51].

MRI is the modality of choice for evaluating an undetermined pelvic mass on ultrasound and also to evaluate the local burden of tumour [52, 53]. Recently Ma et al. [48] have outlined the use of MRI for differentiating PFTC from epithelial ovarian cancer (EOC). According to their assessment of MRI features, the characteristic appearance of PFTC was a relatively small, tubular-shaped (or sausage-shaped) mass, with homogenous signal, low signal intensity on T1 weighted images (T1WI), high signal intensity on T2 weighted images (T2WI), mild to moderate enhancement, and hydrosalpinx or intrauterine fluid. Ma et al. identified tubular (sausage) shape, hydrosalpinx, and the presence of intra-uterine fluid as the most specific direct and indirect signs of PFTC. The combination of an adnexal mass with at least one of the former features yields a high diagnostic accuracy [48]. 
Table 12013 FIGO staging classification for cancer of the ovary, fallopian tube, and peritoneum

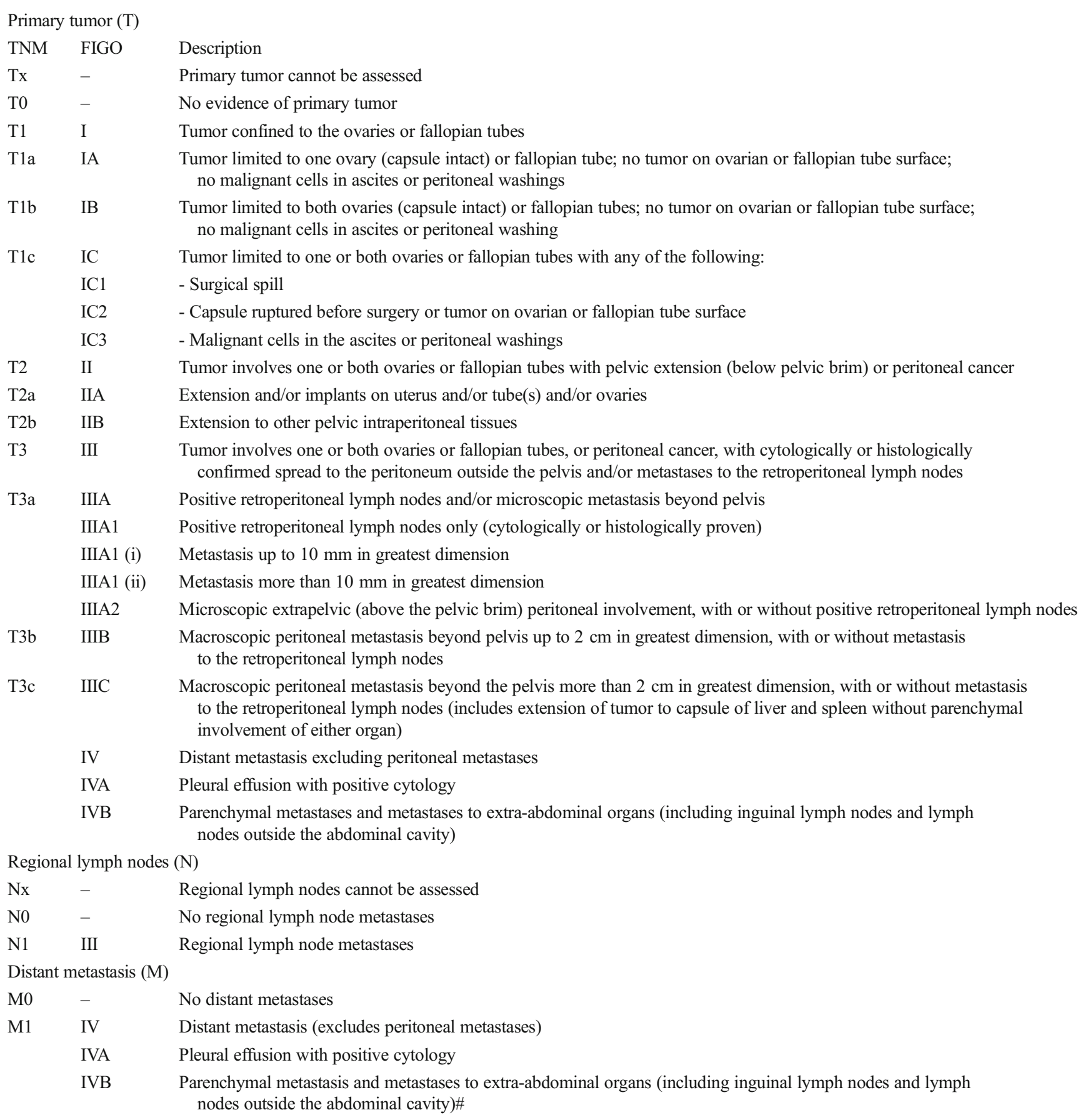

Adapted from Prat J (2014) Staging Classification for Cancer of the Ovary, Fallopian Tube, and Peritoneum. Int J Gynaecol Obstet 124:1-5 (reference 42). (*) Dense adhesions with histologically proven tumour cells justify upgrading to stage II. (\#) Transmural bowel infiltration or umbilical deposit are stage IVB

\section{Anatomy of the fallopian tubes on MRI}

The normal fallopian tubes are usually not visualized on pelvic MRI. In the presence of intraperitoneal fluid, they may be seen as paired thin structures, extending from the ovaries to the uterine cornua, in the superior edge of the broad ligament [2]. They extend for about $10-12 \mathrm{~cm}$ although they will appear shorter due to being convoluted on cross-sectional imaging. The fallopian tubes are divided into four portions, the intramural/interstitial on the medial end, the isthmus, the ampulla, and the infundibulum at the lateral fimbriated end [26] (Fig. 1). 


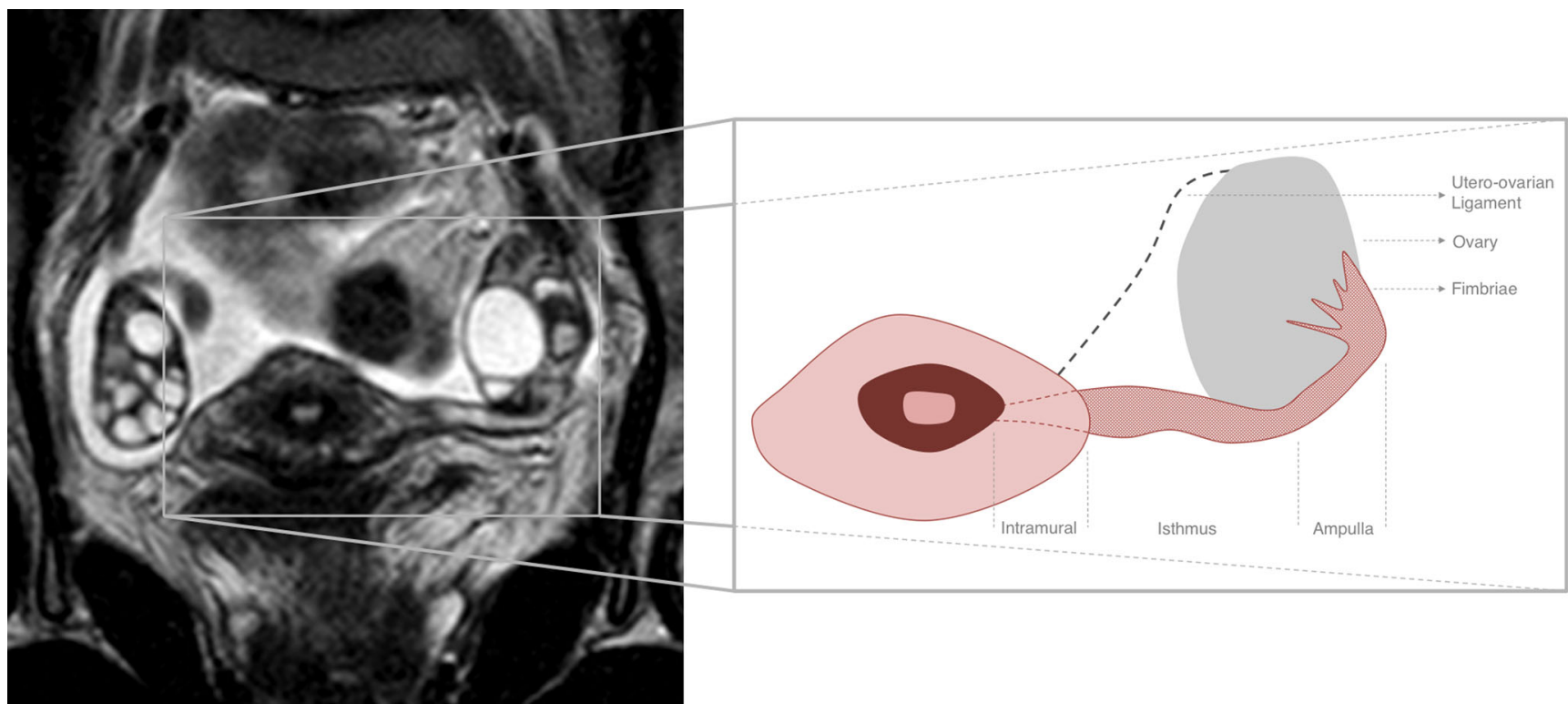

Fig. 1 Thirty-two-year-old female imaged for other purposes. T2WI coronal oblique section through the pelvis showing a normal uterus, both ovaries, and the full length of the left fallopian tube. On the right

hand side of the image, an illustration of the fallopian tube anatomy can be observed, based on the MR image see on the left, showing the four segments of the tube: intramural, isthmus, ampulla, and fimbriae

Fig. 2 Serous PFTC in a 63-yearold female, presenting with pelvic pain. a Axial T2WI through the pelvis, showing "sausage"shaped mass (asterisk). Incomplete folds (red arrow) and the "waist" sign (white arrows), are in favour of a fallopian tube mass; b Sagittal T2WI with the same solid mass (asterisk), inside a structure with well-defined walls (white arrowhead), the "waist" sign (white arrows), incomplete folds (red arrow), and the "synechiae" sign (red arrowhead) with strands of tissue floating in the fluid-filled fallopian tube; c Coronal T2WI, with the "sausage"-shaped mass (asterisk) folded upon itself, as depicted by the portions of wall visible inside the mass (red arrow), which approximates the "spoke-wheel" sign, usually better depicted when the mass is predominantly cystic. Once again, the "synechiae" sign can be observed (red arrowhead); $\mathbf{d}$ T1WI, fat-saturated, post-contrast image, showing the enhancement of the solid portions of the mass. Courtesy of Dra. Rosana Santos
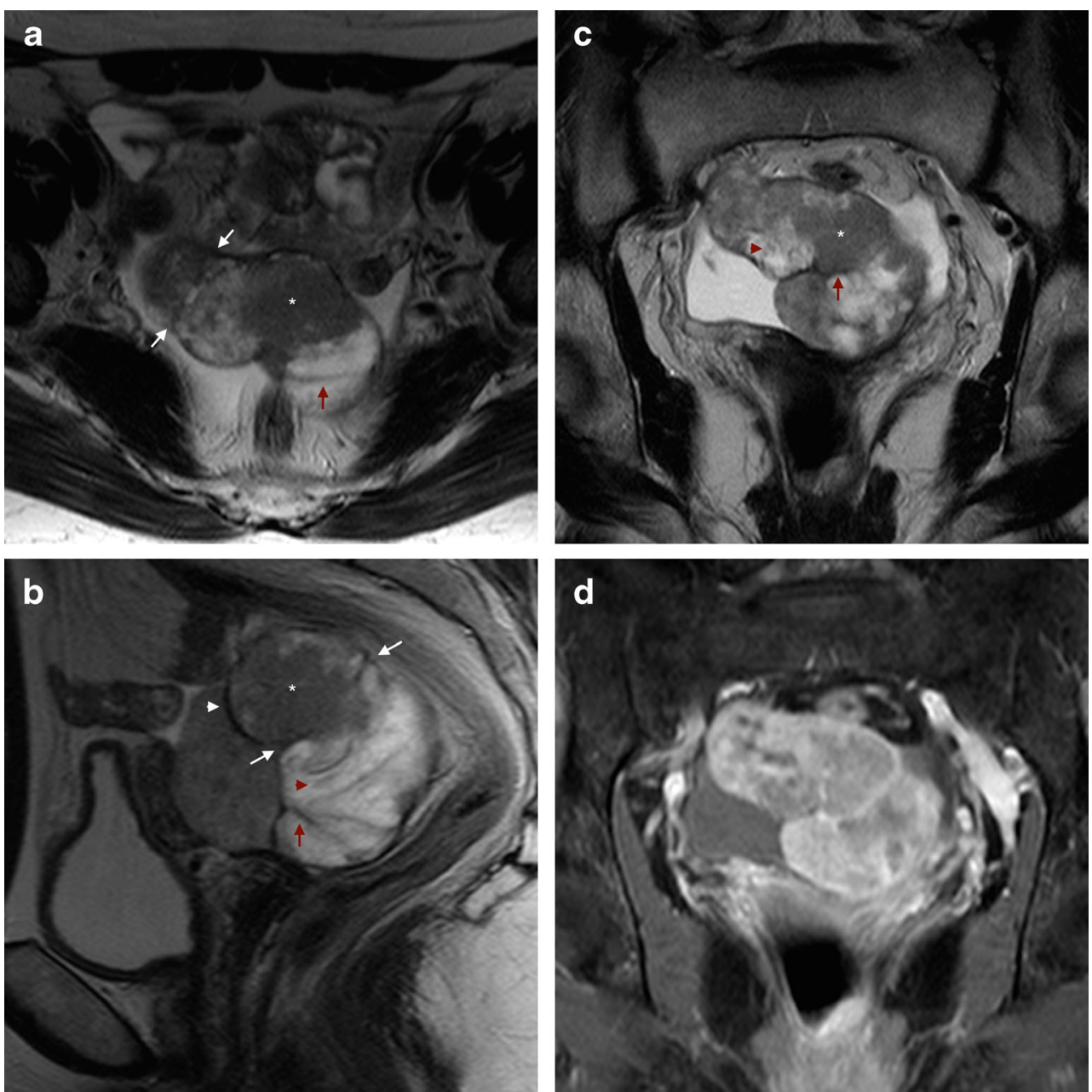
Fig. 3 Serous PFTC in a 78-yearold female, presenting with postmenopausal bleeding and pelvic pain. a Sagittal T2WI showing a solid mass (asterisk) with well-defined walls (white arrow), incomplete folds (red arrow), and an incidental cystic lesion corresponding to a cystadenofibroma (blue arrowhead); b Axial T2WI showing the ovarian cystadenofibroma, posterior to the mass (blue arrowhead); c Axial DWI with the same solid mass showing restriction to diffusion; $\mathbf{d}$ Axial T1WI, fat-saturated, contrast-enhanced image, showing contrast uptake by the mass, with central necrosis
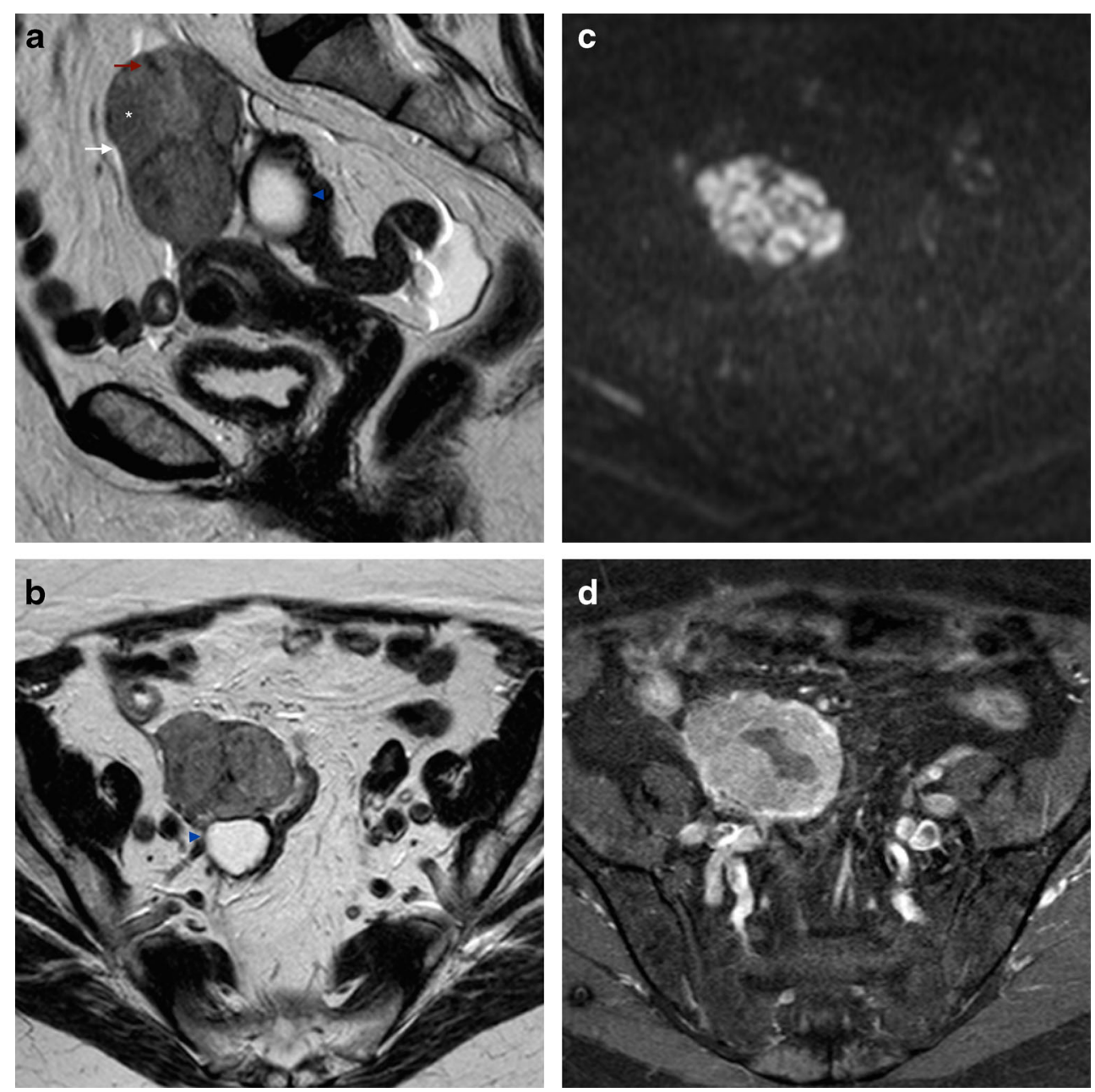

\section{Tubular/sausage-shaped mass}

The tubular nature of these paired organs is seen behind the sausage-shaped appearance when they are filled with solid tumour (Fig. 2). This is one of the most specific signs seen in PFTC, particularly in the presence of contrast enhancement of the mass (Figs. 2d and 5d). The solid tumour may have variable $\mathrm{T} 1$ hypointensity and $\mathrm{T} 2$ hyperintensity and show a high signal in diffusion weighted imaging (DWI) (Fig. 5). Other pathologies can present with hydrosalpinx and solid component, such as a tubo-ovarian abscess, and differentiation from malignancy can be challenging.

\section{Hydrosalpinx}

Hydrosalpinx forms in PFTC both due to the copious amounts of fluid produced by the tumour and to the partial obstruction of the tubes. This leads to tubular distention and can be easily observed in T2WI sequences. Frequently, one of the ends of the tube is patent, leading to decompression of the tubal obstruction with discharge and consequent shrinkage of the pelvic mass, resulting in a temporary resolution of symptoms, as described in the clinical presentation. This variability of size and shape of the mass can be seen in serial imaging. MRI can easily detect the presence of hydrosalpinx, which usually appears as a cystic, tubularshaped, convoluted mass, with well-defined walls. The dilated tubes contain incomplete plicae or folds, producing its convoluted appearance, either seen as the "waist sign" (Fig. 2), causing focal constriction of the tubular structure, or as the "beak sign" (Figs. 3a and 4a), reflecting an acute angular contour, which would not be seen on a regular tubular or round structure [25]. This is distinct from the "beak sign" described as an indicative feature of an intra-ovarian lesion [54]. When a fallopian tube is extremely dilated by fluid, it may fold upon itself to produce the "cogwheel sign", which may be indistinguishable from an ovarian 

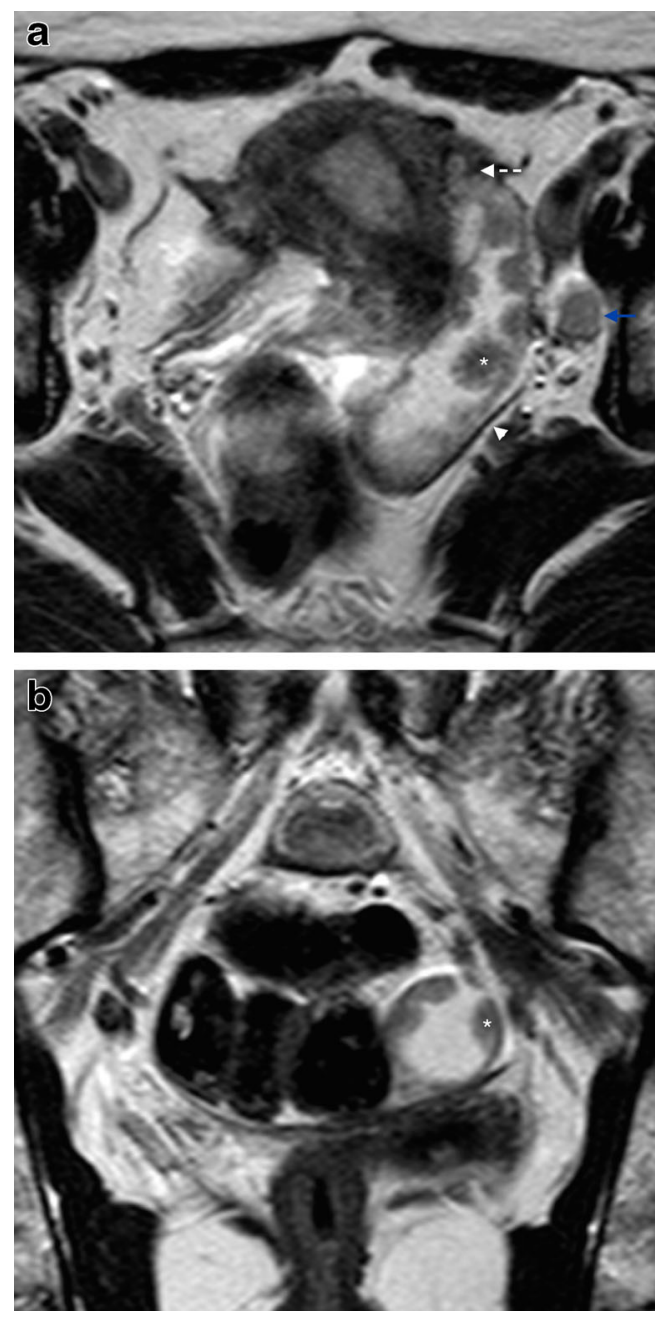

Fig. 4 Serous PFTC in a 58-year-old female, presenting with postmenopausal bleeding. a Axial T2WI showing a left hydrosalpinx with welldefined walls (arrowhead), mural nodules (asterisk), the "beak sign" (dashed arrow), and an enlarged left obturator lymph node (blue arrow); b Coronal T2WI showing the same mural nodules (asterisk) inside the fluid-distended left fallopian tube, in transverse section

neoplasm [2]. Occasionally, the "synechiae sign", consisting of fine strands running across the lumen, can also be seen in dilated fallopian tubes [25] (Fig. 2b). Another MRI sign associated with the fallopian tubes is the "amorphous shading sign", corresponding to the loss of signal intensity of the fluid filled tubes from T1WI to T2WI. The high viscosity and the presence of protein and iron due to recurrent haemorrhage, similar to what is seen in ovarian lesions, can explain this phenomenon [55]. The patency of the fallopian tube can lead to discharge of fluid into either the intra-uterine or the peritoneal cavities. However, ascites is not a specific sign of PFTC and is frequently present in ovarian cancer. Intra-uterine fluid, on the other hand, is specific of PFTC and it can occur in up to $30 \%$ of cases [48] (Figs. $4 b$ and 5).
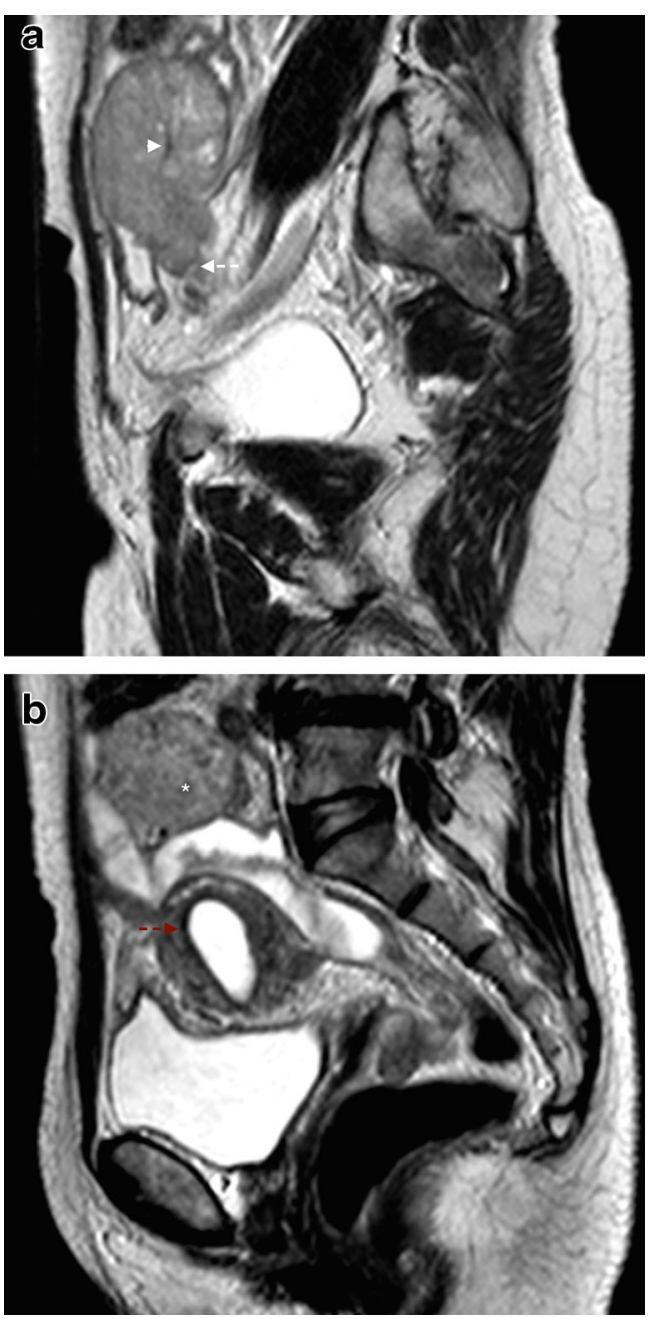

Fig. 5 Serous PFTC in a 64-year-old female, presenting with abdominal pain. a Sagittal T2WI showing a "sausage"-shaped solid mass, folded upon itself as shown by the portion of Fallopian tube wall on the centre of the mass (arrowhead), and again the "beak" sign is depicted (dashed arrow); b Sagittal T2WI where the mass can still be seen (asterisk), together with an important sign in PFTC: hydrometra (red dashed arrow)

\section{Conclusions}

PFTC can be suspected on MRI when specific fallopian-tube related signs are present. However, the likelihood to present when already spread to the ovaries or peritoneum makes this diagnosis challenging, even for the experienced radiologist.

PFTC has a variety of MRI findings, which make it difficult to suspect its origin based solely on imaging. However, radiologists can be more suspicious in the presence of features related to fallopian tube disease, such as a relatively small, tubular-shaped (or sausage-shaped) mass, with homogenous signal, low signal intensity on T1WI, high signal intensity on T2WI, with mild to moderate enhancement, and associated hydrosalpinx or intrauterine fluid. 
Singh's [40] proposal for the histopathological diagnosis of high grade serous carcinoma, suggests assigning the fallopian tube as the primary site whenever there is a fallopian tube mass, even in the presence of other larger concomitant tumours. Radiologists should feel more confident in suspecting PFTC, and reporting it on their differential, in the presence of an adnexal mass associated to one or more of the three more specific signs (tubular-shaped mass, hydrosalpinx, and intrauterine fluid accumulation), particularly in earlier stage disease and when the ovaries can be identified as normal.

Open Access This article is distributed under the terms of the Creative Commons Attribution 4.0 International License (http://creativecommons.org/licenses/by/4.0/), which permits unrestricted use, distribution, and reproduction in any medium, provided you give appropriate credit to the original author(s) and the source, provide a link to the Creative Commons license, and indicate if changes were made.

\section{References}

1. Pectasides D (2006) Fallopian tube carcinoma: a review. Oncologist 11:902-912

2. Rezvani M, Shaaban AM (2011) Fallopian tube disease in the nonpregnant patient. RadioGraphics 31:527-548

3. Shaaban AM, Rezvani M (2012) Imaging of primary fallopian tube carcinoma. Abdom Imaging 38:608-618

4. Alvarado-Cabrero I, Stolnicu S, Kiyokawa T et al (2013) Carcinoma of the fallopian tube: results of a multi-institutional retrospective analysis of 127 patients with evaluation of staging and prognostic factors. Ann Diagn Pathol 17:159-164

5. Riska A, Leminen A, Pukkala E (2003) Sociodemographic determinants of incidence of primary fallopian tube carcinoma, Finland 1953-97. Int J Cancer 104:643-645

6. Goodman MT, Shvetsov YB (2009) Incidence of ovarian, peritoneal, and fallopian tube carcinomas in the United States, 19952004. Cancer Epidemiol Biomarkers Prev 18:132-139

7. Nik NN, Vang R, Shih I-M, Kurman RJ (2014) Origin and pathogenesis of pelvic (ovarian, tubal, and primary peritoneal) serous carcinoma. Annu Rev Pathol Mech Dis 9:27-45

8. Chan A, Gilks B, Kwon J, Tinker AV (2012) New insights into the pathogenesis of ovarian carcinoma. Obstet Gynecol 120:935-940

9. Dubeau L, Drapkin R (2013) Coming into focus: the nonovarian origins of ovarian cancer. Ann Oncol 24:viii28-viii35

10. Reitsma W, de Bock GH, Oosterwijk JC et al (2013) Support of the "fallopian tube hypothesis" in a prospective series of risk-reducing salpingo-oophorectomy specimens. Eur J Cancer 49:132-141

11. Dietl J (2013) Revisiting the pathogenesis of ovarian cancer: the central role of the fallopian tube. Arch Gynecol Obstet 289:241-246

12. Zheng W, Fadare O (2012) Fallopian tube as main source for ovarian and pelvic (Non-endometrial) serous carcinomas. Int J Clin Exp Pathol 5(3):182-186

13. Seidman JD, Zhao P, Yemelyanova A (2011) "Primary peritoneal" high-grade serous carcinoma is very likely metastatic from serous tubal intraepithelial carcinoma: assessing the new paradigm of ovarian and pelvic serous carcinogenesis and its implications for screening for ovarian cancer. Gynecol Oncol 120:470-473

14. Callahan MJ, Crum CP, Medeiros F et al (2007) Primary fallopian tube malignancies in BRCA-positive women undergoing surgery for ovarian cancer risk reduction. J Clin Oncol 25:3985-3990
15. Erickson BK, Conner MG, Landen CN (2013) The role of the fallopian tube in the origin of ovarian cancer. Am J Obstet Gynecol 209:409-414

16. Vang R, Shih I-M, Kurman RJ (2012) Fallopian tube precursors of ovarian low- and high-grade serous neoplasms. Histopathology 62:44-58

17. Berek JS, Crum C, Friedlander M (2012) Cancer of the ovary, fallopian tube, and peritoneum. Int J Gynaecol Obstet 119(Suppl 2):S118-S129

18. Chêne G, Dauplat J, Radosevic-Robin N et al (2013) The fallopian tube odyssey: from the ovary to the tube. About high-grade serous ovarian carcinoma. Bull Cancer 100:757-764

19. Horn L-C, Kafkova S, Leonhardt K et al (2013) Serous Tubal in Situ Carcinoma (STIC) in primary peritoneal serous carcinomas. Int J Gynecol Pathol 32:339-344

20. Lau H-Y, Chen Y-J, Yen M-S et al (2013) Primary fallopian tube carcinoma: a clinicopathologic analysis and literature review. J Chin Med Assoc 76:583-587

21. Slanetz PJ, Whitman GJ, Halpern EF et al (1997) Imaging of fallopian tube tumors. AJR Am J Roentgenol 169:1321-1324

22. Vyas MN (2013) Bilateral primary fallopian tube carcinoma with the classical clinical features: a case report. JCDR. doi:10.7860/ JCDR/2013/4518.2894

23. Kawakami S, Togashi K, Kimura I et al (1993) Primary malignant tumor of the fallopian tube: appearance at $\mathrm{CT}$ and MR imaging. Radiology 186:503-508

24. Outwater EK, Siegelman ES, Chiowanich P et al (1998) Dilated fallopian tubes: MR imaging characteristics. Radiology 208:463-469

25. Ghattamaneni S, Bhuskute NM, Weston MJ, Spencer JA (2009) Discriminative MRI features of fallopian tube masses. Clin Radiol 64:815-831

26. Kim MY, Rha SE, Oh SN et al (2009) MR imaging findings of hydrosalpinx: a comprehensive review1. RadioGraphics 29:495-507

27. Haratz-Rubinstein N, Russell B, Gal D (2004) Sonographic diagnosis of fallopian tube carcinoma. Ultrasound Obstet Gynecol 24: 86-88

28. Kalampokas E, Kalampokas T, Tourountous I (2013) Primary fallopian tube carcinoma. Eur J Obstet Gynecol Reprod Biol 169(2):155-161

29. Riska A, Martinsen JI, Kjaerheim K et al (2011) Occupation and risk of primary fallopian tube carcinoma in Nordic countries. Int J Cancer 131:186-192

30. Ajithkumar TV, Minimole AL, John MM, Ashokkumar OS (2005) Primary fallopian tube carcinoma. Obstet Gynecol Surv 60:247-252

31. Horng HC, Teng SW, Huang BS et al (2014) Primary fallopian tube cancer: domestic data and up-to-date review. Taiwan J Obstet Gynecol 53(3):287-292

32. Tentugal CN, Cunha TM, Félix A (2013) Fallopian tube carcinoma: pearls and pitfalls. EPOS - Elect Present Online Syst. doi:10.1594/ ecr2013/C-0543

33. Caldeira JP, Cunha TM (2008) Fallopian tube carcinoma. Eurorad European Radiology Online Database - "Certified Radiological Teaching Cases" \{Online\} URL: http://www.eurorad.org/case.php doi: 10.1594/EURORAD/CASE.7075

34. Hefler LA, Rosen AC, Graf AH et al (2000) The clinical value of serum concentrations of cancer antigen 125 in patients with primary fallopian tube carcinoma: a multicenter study. Cancer 89:1555-1560

35. WHO Classification of Tumours of Female Reproductive Organs (2014). In: Kurman RJ, Carcangiu ML, Herrington CS, Young RH, World Health Organization, 4th Edn. Lyon 
36. Gadducci A, Landoni F, Sartori E et al (2001) Analysis of treatment failures and survival of patients with fallopian tube carcinoma: a Cooperation Task Force (CTF) study. Gynecol Oncol 81:150-159

37. Hu CY, Taymor ML, Hertig AT (1950) Primary carcinoma of the fallopian tube. Am J Obstet Gynecol 59:58-67, illust

38. Sedlis A (1961) Primary carcinoma of the fallopian tube. Obstet Gynecol Surv 16:209-226

39. Sedlis A (1978) Carcinoma of the fallopian tube. Surg Clin North Am 58:121-129

40. Singh N, Gilks CB, Wilkinson N, McCluggage WG (2014) Assignment of primary site in high-grade serous tubal, ovarian and peritoneal carcinoma: a proposal. Histopathology 65:149-154

41. Aich RK, Dasgupta S, Chakraborty B et al (2012) Primary fallopian tube carcinoma with metastasis in the contralateral ovary. J Indian Med Assoc 110:494-5, 498

42. Prat J (2014) Staging classification for cancer of the ovary, fallopian tube, and peritoneum. Int J Gynaecol Obstet 124:1-5

43. Kosary C, Trimble EL (2002) Treatment and survival for women with fallopian tube carcinoma: a population-based study. Gynecol Oncol Aug 86(2):190-191

44. di Re E, Grosso G, Raspagliesi F, Baiocchi G (1996) Fallopian tube cancer: incidence and role of lymphatic spread. Gynecol Oncol 62: 199-202

45. Morgan R. NCCN Clinical practice guidelines in oncology. Ovarian cancer. Version 3.2014

46. Usach I, Blansit K, Chen L-M et al (2015) Survival differences in women with serous tubal, ovarian, peritoneal, and uterine carcinomas. Am J Obstet Gynecol 212:188.e1-6

47. Moore KN, Moxley KM, Fader AN et al (2007) Serous fallopian tube carcinoma: a retrospective, multi-institutional case-control comparison to serous adenocarcinoma of the ovary. Gynecol Oncol 107:398-403

48. Ma FH, Cai SQ, Qiang JW et al (2014) MRI for differentiating primary fallopian tube carcinoma from epithelial ovarian cancer. J Magn Reson Imaging. doi:10.1002/jmri.24740

49. Kol S, Gal D, Friedman M, Paldi E (1990) Preoperative diagnosis of fallopian tube carcinoma by transvaginal sonography and CA125. Gynecol Oncol 37:129-131

50. Arko D, Žegura B, Virag M et al (2014) Preoperative diagnosis of fallopian tube malignancy with transvaginal color doppler ultrasonography and magnetic resonance imaging after negative hysteroscopy for postmenopausal bleeding. Coll Antropol 38: $1047-1050$

51. Hosokawa C, Tsubakimoto M, Inoue Y, Nakamura T (2006) Small fallopian tube carcinoma with extensive upper abdominal dissemination: a case report. AJR Am J Roentgenol 186:1046-1050

52. Spencer JA, Forstner R, Cunha TM et al (2010) ESUR guidelines for MR imaging of the sonographically indeterminate adnexal mass: an algorithmic approach. Eur Radiol 20:25-35

53. Anthoulakis C, Nikoloudis N (2014) Pelvic MRI as the "gold standard" in the subsequent evaluation of ultrasoundindeterminate adnexal lesions: a systematic review. Gynecol Oncol 132:661-668

54. Nishino M, Hayakawa K, Minami M et al (2003) Primary retroperitoneal neoplasms: CT and MR imaging findings with anatomic and pathologic diagnostic Clues1. RadioGraphics 23:45-57

55. Lopes Dias J, Veloso Gomes F, Lucas R N, Cunha TM (2014) Conference paper: the shading sign: is it exclusive of endometriomas? RSNA E351: doi: 10.13140/2.1.4502.3526 